\title{
Participation and Communication in the Time of Social Media: A Chimera or an Opportunity
}

\section{Andrea Volterrania}

\begin{abstract}
Communication and participation immediately present themselves as a combination capable of developing mutual symbiosis and reinforcement. However, it would be superficial to deduce that the ease of communicating through social media has solved the problem of participation. Instead, it is necessary to start from the awareness of the problems of this binomial in order to attempt to follow virtuous paths and avoid blind alleys. The paper presents the research/action carried out in several civic participation experiences, both real and virtual, promoted by NGOs and the local public administrations of several Italian cities (Verona, Messina, Bolzano, and Rome). The results highlight the opportunities and problems of participation through social media. Specifically, those opportunities are: (1) the possibility of involving a larger number of people who could not participate without social media; (2) the sharing not only of the results of the participation but also the processes behind the participation allows for an active protagonism of the people; and (3) the care of the real and virtual places of participation facilitates the active involvement of people. On the other hand, the problems are: (1) the lack of awareness of online media space generates a redundancy in communication; (2) the difficulty of emerging in the online public media space causes disaffection and disinterest; and (3) the scarce capability to be producers of online media content generates a fake participation.
\end{abstract}

\section{Keywords}

Participation, social media, citizens

Monster of divine origin, lion's head, goat's chest and dragon's tail; and from the mouth horrific blazes vomiting of fire Homer, The Iliad

\section{WHY PARTICIPATION AND COMMUNICATION}

The Chimera was viewed as a sort of symbol of change, albeit with a negative meaning as a distorted image of the trinity. Over the centuries, the Chimera has finally evolved into a symbol of illusions, risky fantasies, and unrealizable and dangerous dreams.

The idea that citizen participation could increase through social media (Jenkins 2006; Jenkins, Ford, and Green 2013; Dahlgren 2009) is, in part, a chimera of the hypermodern and contemporary society (Colombo 2013; Couldry and Hepp 2017; Floridi 2016).

The issue of participation has always been at the center of thought of those who cared about the full involvement of citizens in the processes of planning,

aUniversity of Rome Tor Vergata, Italy

\section{Correspondent Author:}

Andrea Volterrani, Department of Enterprise Engineering, University of Rome Tor Vergata, Via Columbia 200137 Rome, Italy 
programming, choice, and government of communities. But, in fact, it often remained in thought only, in good intentions, and rarely had the opportunity to develop concretely in daily practices.

More recently, with the diffusion of social media, the idea of easy citizen participation has come back strongly to the fore. What we would like to discuss here is if and how contemporary communication in all its expressions is connected to participation.

\section{INDIVIDUAL OR COLLECTIVE}

An initial reflection. Is participation individual or collective? It is an important element if connected to communication. Everyone participates as an individual in something (an activity, project, event, service, organization, etc.), and each can choose to remain isolated in their activity or involve other people. The difference lies, above all, in the social consequences of participation; that is, in the ability to construct a more or less broad and more or less continuous involvement over time. Time and involvement are variables that, when they remain at the individual level, do not allow for the growth of groups, communities, and real or virtual territories; they are, however, likely to create profound social and cultural inequalities between people. If someone participates individually in a project for the development of their neighborhood alone, they will certainly acquire knowledge and skills that will increase their individual abilities and sensibilities. But they will not be able to promote real change in that neighborhood because individual participation alone has no social consequences. It seems an obvious statement, but in organizational everyday experiences, losing the sense of participation is lost.

\section{THE PLACES OF PARTICIPATION}

Real or virtual? With the characteristics of contemporary communication, the dilemma does not arise because we can activate a participatory process in the territory (such as how a public space should be managed for the younger generations), continue on social media to discuss the issue, even with those who could not be present; and then return to the territory with a higher number of participants and a greater awareness of the necessary actions to be undertaken. There is a continuity between the real and the virtual that has been established in the daily life experiences of many people, even among those who are not digital natives. Perhaps there still are some obstacles to not fully grasping this fluidity, but surely many have had experiences of real actions that were subsequently transferred online and then came back strongly into reality, either positively or negatively, including or excluding people.

The places of participation have become ubiquitous in on- and off-line territories (Boccia Artieri et al. 2017). Ubiquitous is because through smartphones and tablets people are able to stay connected to almost any part of the territories in which they live, and which, in turn, have multiplied through the exponential growth of the networks of relationships in which the inhabitants participate. Certainly there are empty spaces in these places, isolations either sought after or suffered by people, groups, and contexts; vulnerabilities that do not allow people to stay in the places of participation. There is a cultural digital divide that is far more important than the digital divide alone (Couldry 2010). Cultural and social inequalities are the first major obstacle to accessing not only online participation sites but also those located off-line. Focus is often placed only on the technological side of the matter, forgetting that the real problem is the social and cultural one: despite having technical skills, we can safely ignore what is potentially interesting and useful for us.

Here then are two important first points: on the one hand, the care and promotion of places of participation and, on the other, the activation of the processes of inclusion for those who can not access them. The care of places means creating the conditions so that 
participation can take place in a simple, conscientious, and respectful manner of each person's time. For example, it is not possible to consider online platforms that only allow access to those possessing sophisticated skills or platforms that offer paths of participation so twisted that even the first orientation is not easy. Moving on to real spaces, another example is the penalizing condition of immovable seating, chairs that are fixed to the ground so that it is not possible to imagine different configurations. The layout design of on- and off-line places of participation is a fundamental skill not easily found in many organizations.

The promotion of places is another important aspect. People are often mistakenly convinced that any place they know must be a well-known place because it is particularly familiar to them. It is a perspective that hinders participation both on- and off-line. Most people do not perceive and do not know the available spaces where they can share opinions on ideas, activities, and projects with others. Communication is central to both care and promotion. Making the places of participation simple and accessible also means sharing communicative processes with potential participants, just as making a place more well-known is strictly connected to the essence of communication.

The inclusive participatory processes are very difficult because they clash with a lack of awareness as well as with a widespread skepticism about the opportunity to participate. Basically, the questions are: Why should I "waste time" participating? What will I get out of it? This is what happens, what we ask ourselves when someone asks us, for example, to write a proposal document or a project idea with others. In addition to Hirschman's (1982) free rider theory, the overcoming of these barriers still passes from communication that, like a vessel that crosses borders, can accompany us from the perception to the change of behavior, and not without difficulty. In other cases, the process of inclusion clashes with a vulnerability of people (cultural, social, and economic), which adds on a problem that is not easily solved. If we want to build a place of participation for vulnerability, we must first work to raise awareness of ourselves and the context to the people we want to involve. The communicative processes that come into play are not strictly connected to participation (which comes later), but to the change of the individual and/or territorial culture. One of the difficulties is that "perception is not bought" and, consequently, not even awareness, which is preliminary. So, where to start?

\section{THE PROTAGONISM IN PARTICIPATION AND COMMUNICATION}

The development of protagonism is one of the possible paths to follow, albeit with many ambivalences. It is not easy to build paths where people are protagonists in the identification of issues and problems, in discussions and decisions, because it is first of all necessary to respect the times, sensitivities, and potentialities of each one. Anyone who has had to deal with groups, for training, not only work-related but also for leisure time, has experienced the sometimes extreme differentiation between people. A resource for the different opinions, points of view, and projects that can be put in place. A sometimes insurmountable problem if the voices do not agree and, above all, if active listening does not take place from the beginning. Fatigue. It is the term that those who pursue participation use to feel better. Because interpersonal and group communication requires a lot of energy from all those who take part, especially if combined with the need to enter into a relationship with someone who does not share our tastes, our passions, our way of understanding the world. There are techniques of facilitation and communication that favor the protagonism in participation (Sclavi 2003), but what is most important is to be able to activate once again a communication process that helps, with time and training, to move from perception to incorporation. The author focuses on training because he believes it is the most important 
practice on a communicative and participatory level. Thinking and then participating are not immediate activities, but rather need to be tried out in protected places before moving on to real and virtual territories. The "participation gyms" can be many: some examples are social sporting activities, groups that act with a limited purpose (e.g., the monthly collective removal of garbage from a neighborhood), the management of neighborhood parties, Whatsapp groups of peers or of parents of students in the same class, etc. All of these are activities lacking the potential to change the world. Indeed, their ability to influence real and virtual communities is very poor, to say the least. However, by taking part in these simple activities, one can learn through the group's communication to become aware of how to participate better, more actively, and with respect for others.

The growth of awareness and development of the skills required in the "participation gyms" then allow one to build and develop participation spaces which have greater social impact and visibility.

\section{THE SHARED AND PARTICIPATED PRODUCTION}

The second path we are going to travel together is that of the shared and participated production of tangible and intangible products. In theoretical literature and through the analysis of contemporary media, the question of production linked to individual consumption has a precise name: prosumers (Jenkins et al. 2013). Each of us is a potential prosumer, in social media especially, but also in the territories and communities in which we live. What does it mean? Normally, we are consumers of content (products, services, and projects) and relationships (real and virtual), but for a decade now, it has also been possible to be producers (real and virtual), or more precisely, producers and consumers at the same time. For example, new media technologies have lowered the access threshold to multimedia productions that can be considered good quality (trailers, moodboards, and individual and/or group storytelling). Another example is the opportunities in territorial communities to construct contexts and spaces that produce small activities and products of embryonic social agriculture or the sharing of spaces for collective hospitality (not only that of the widespread hotel), where families or single people promote informal reception to tourists, travelers, and refugees. They are all examples where we become something more: citizenusers, producers, and consumers of inclusive and participated citizenship. One aspect, however, which is fundamental in our reasoning is that production, is not only shared but is built in a participatory way. If it were not, we would have reproduced the same mechanisms of transmission of content (from a source to a recipient) that so much damage has been produced and produced for a real dissemination of knowledge and experiences. Participated production of knowledge and experience is not just a slogan, but also a style that starts from the ideation and design discussed and participated with those who collaborate or could collaborate, with those who are enjoying or potentially enjoying, and with those who could contribute to the actual realization. A strong involvement (engagement) that is thought of and implemented from the beginning or that is activated during construction, but which tends to both enhance the protagonism at the start of any shared and participated production activity and to involve all those potentially interested. However, examples of this kind are not found in the landscape of Italy because Italians are still attached to the idea that it is too difficult, too tiring, to be able to pursue this path with conviction. Nevertheless some prevention activities that aim at increasing social resilience are trying to follow this path. Some examples include the "I do not take risks" campaign promoted by the Italian National Civil Protection Department, which was created in collaboration with voluntary organizations and involved a strong activation of the territorial communities in verifying and increasing the skills to be 
used in case of natural disasters (earthquake or flood), and the involvement in and the protagonism of the elderly and disabled in the construction of a data base to be used in emergencies and to be updated periodically online by the elderly and disabled themselves with the assistance of both public and voluntary services. Other examples include experiences of community development that were started in Verona owing to a collaboration between local authorities and social cooperation, and in the Messina area of Sicily, where, with great environmental difficulties, some young people "discover" their territory through the eyes of their peers in neighborhoods and degraded areas thanks to the support of the local service center for volunteering. As these examples demonstrate, small-scale experiences of participated production of knowledge are slowly entering the Italian scene. And communication (interpersonal, mediated, and complex) is at the center of each of these activities. But what is the risk?

\section{HOW TO GIVE CONTINUITY TO PARTICIPATION (AND COMMUNICATION)}

The problem we encounter most often is that of continuity in participation. Actions often start off with great enthusiasm and then, after a shorter period of time, become less participated in. Sometimes it is due to the first difficulties that are encountered or the demobilization of participants who do not see immediate and rewarding results or, again, the participants lose interest because they have moved on to other themes, other involvements, or other groups.

The third reason, therefore, for why participation is not a chimera is continuity in participatory and communicative action. Given that there is not a list of standarized rules that apply to all situations, some indications and reflections could be useful. First of all, it is essential that the starting point does not rest on the basis of enthusiasm and emotion. They are great resources for participation, but they become quickly exhausted in the space of a season because they often do not incorporate the meanings and the sense of participation. Emotionality is difficult to manage because it often represents the first strong push that causes an activity, project, or initiative to take off. But it is important to give space to reflexivity, balancing between the emotion of the moment and the prospect of future growth, between involvement and detachment (Elias 1987). Try to imagine a different participation from that of the emotional one during the interventions that follow a natural disaster or any other kind of crisis, you will have found an initial access to the third road.

Another aspect to consider is, obviously, the close link with communication. Any type of participation is likely to constitute a point of reference for a small group of people, but it is incapable of enlarging and/or including other people with the same protagonism of the first arrivals. It is also the problem faced by many organizations where the inclusion of new participants is cumbersome, if not hindered. In this case, it is organizational communication that could support organizations in giving continuity to participatory action, linking internal and external communication with internal and external participation. It means thinking of open, democratic organizations that are not only concerned with their own survival but capable of developing meaning for their members (members, citizens, and users) as well as for those who are non-members. It means abandoning the idea that participation and communication are activities that are carried out just because "we have nothing else to do". Instead, they are important objectives that, in addition to needing continuity, give continuity to the organization itself and foreshadow the future of our communities.

\section{FAKE PARTICIPATION}

Participation can not be an end in itself. Occasionally some participation and communication techniques are presented as the final goal to be achieved. It is a huge 
mistake that makes us lose sight of the fact that participation makes sense for a goal, for the realization of an activity, to change a social context, a neighborhood, a community, or a city; and to develop and share new ideas-all activities involving participation and communication as fundamental agents of support, facilitation, and sharing, but not substitution.

When this happens, it is often the individual and collective happiness of the moment that takes the upper hand and those who participate mistake this for real participation. But when the activities inevitably return to the routine, then this explosive happiness fades, leaving room for reflection and the sense of participation.

The other great mystification of participation is that which we find within social media, where we think that through actions which reward visibility and presence (for example, with the number of likes on Facebook, Twitter, or Instagram), we are participating in important events. Our protagonism is only a façade because it does not affect, other than superficially, attitudes, ideas, and behaviors that are rooted in our daily lives. Even when we believe we are expressing a more important preference (for example, an electronic vote on something), we are simply validating choices made by someone who has built and manages a framework where all of this takes place (Couldry 2010; Colombo 2013).

Finally, fake participation is that which is carried out daily in almost all organizations (which, in recent times, pop up practically every day!) that develop actions of social cohesion, community development, mutuality, and social innovation without worrying in the least if all this has been thought about, shared, communicated, and, above all, decided on within the context (communities, neighborhoods, groups of citizens), which will then benefit from consequent activities and projects. On many occasions, everything is presented as a great idea of participation and communication and as a good practice to follow, but in reality nothing happens at all. Perhaps this last fake is only a bubble deriving from the moment of transition and transformation that we are experiencing, but, unfortunately, it risks damaging good participation and good communication.

\section{CONCLUSIONS}

At the end of this brief reflection, the author once again dwells on the links between participation and communication. What follows is a brief, non-exhaustive, and non-binding list of suggestions.

Participation and communication work on sharing content, meanings, and symbols. Definitely abused but still little practiced in the organizational and territorial realities that can neither think nor plan nor even do, really and/or symbolically, sharing. Both foresee the protagonism of human beings. Participation is by people alone and in groups, territorial communities, organizations, and movements that embody ideas, projects, and actions. In communication, it is the human being, as taught by the School of Palo Alto, which can not fail to communicate as such. Both, one in function of the other and vice versa, place relations between people as the engine for development at the center. Without relationships, there can be neither participation nor communication. Both, if acted to the end, are changes: of people, of the individual and collective imagination, and of individual, community, and territorial reality. Finally, both are projected towards the future starting from their roots in the present. Participation builds from people who is not yet there and who, perhaps, could be. Communication, if it is not that of the market, builds new imaginaries capable of building new future horizons and new visions.

Sharing, protagonism of the actors, relationality, change, future. It is from these five words that a discussion can start on how to make participation and communication in the time of social media an opportunity for all organizations, and not just a chimera. 


\section{Funding}

The author is grateful for research support from the National Institute on Drug Abuse (R01DA13459) and the Centers for Disease Control and Prevention (R49 CCV423114).

\section{References}

Boccia Artieri, G., L. Gemini, F. Pasquali, S. Carlo, M. Farci, and M. Pedroni. 2017. Fenomenologia dei social network (Phenomenology of Social Networks). Milano: Guerini Scientifica.

Colombo, F. 2013. Il potere socievole (Sociable Power). Milano: Bruno Mondadori.

Couldry, N. 2010. Why Voice Matters. Culture and Politics After Neoliberalism. London: Sage Publications.

Couldry, N. and A. Hepp. 2017. The Mediated Construction of Reality. Cambridge: Polity.

Dahlgren, P. 2009. Media and Political Engagement: Citizens, Communication and Democracy. London: Cambridge University Press.

Elias, N. 1987. Involvement and Detachment. New York:
Blackwell.

Floridi, L. 2016. The Fourth Revolution: How the Infosphere Is Reshaping Human Reality. London: Oxford University Press.

Hirschman, O. 1982. Shifting Involvements: Private Interest and Public Action. New York: Princeton University Press.

Jenkins, H. 2006. Convergence Culture: Where Old and New Media Collide. New York: New York University Press.

Jenkins, H., S. Ford, and J. Green. 2013. Spreadable Media: Creating Value and Meaning in a Networked Culture. New York: New York University Press.

Sclavi, M. 2003. Arte di ascoltare e mondi possibili (Art of Listening and Possible Worlds). Milano: Bruno Mondadori.

\section{Bio}

Andrea Volterrani, Ph.D., professor and researcher, Department of Enterprise Engineering, University of Rome Tor Vergata, Italy; research fields: nonprofit organizations communication, participation, social media, social resilience, and community development. 